\title{
DENSIDADE E UMIDADE DO SOLO EM SISTEMAS AGROFLORESTAIS
}

\author{
Raylana Rodrigues da Fonseca'; Kamila Stephanie Sousa Barbosa²; Kássia Elen de Jesus ${ }^{3}$; Luara \\ Marques da Silva4; Nathalia Thuanny Barros Sousa ${ }^{5}$; Geny Rocha da Silva6; Daniela Pauletto; ${ }^{7}$ Maisa \\ Yurika Ferreira Otake ${ }^{8}$. \\ ${ }^{1}$ Universidade Federal do Oeste do Pará, Santarém, Pará, Brasil, raylanafonseca@gmail.com \\ 2 Universidade Federal do Oeste do Pará, Santarém, Pará, Brasil, kamilastephanie7@gmail.com \\ 3 Universidade Federal do Oeste do Pará, Santarém, Pará, Brasil, kassiaelen95@hotmail.com \\ 4 Universidade Federal do Oeste do Pará, Santarém, Pará, Brasil, luara_marques.s@hotmail.com \\ ${ }^{5}$ Universidade Federal do Oeste do Pará, Santarém, Pará, Brasil, nathaliabarrosflı@gmail.com \\ 6Universidade Federal do Oeste do Pará, Santarém, Pará, Brasil, geny.silva14@gmail.com \\ ${ }^{7}$ Universidade Federal do Oeste do Pará, Santarém, Pará, Brasil, danielapauletto@hotmail.com \\ ${ }^{8}$ Embrapa, Macapá, Amapá, Brasil, maisa.otake@hotmail.com
}

RESUMO: Os sistemas agroflorestais (SAFs) são sistemas integrados constituídos por espécies agrícolas e florestais, os quais buscam manter as condições naturais do solo. Deste modo, o trabalho objetivou avaliar e comparar dois atributos físicos do solo, densidade e umidade, em diferentes sistemas agroflorestais. $O$ estudo foi realizado no município de Monte Alegre - Pará em uma coleta pontual em junho de 2017. As amostras de solo foram coletadas utilizando o método do anel volumétrico, considerando-se a profundidade de 0-5 cm no solo para a determinação da densidade e umidade. As análises foram realizadas na área de triagem do viveiro da Universidade Federal do Oeste do Pará. O delineamento experimental foi inteiramente casualizado com seis tratamentos e cinco repetições. Os dados foram submetidos ao teste de normalidade, e com base neste realizou o teste não paramétrico de Kruskal Wallis para a densidade, devido a não normalidade dos dados, e o teste de Tukey para os dados de umidade. A densidade encontrada nos SAFS estudados variou de 0,7 a 1,2 g. $\mathrm{cm}^{-3}$, sendo assim considerados solos em condições normais, não apresentando níveis de compactação. Dentre os sistemas estudados, o SAF 4 apresentou maior nível de densidade e menor teor de umidade e o SAF 3 apresentou melhores condições com maior valor de umidade e menor de densidade. Aparentemente o histórico anterior ao plantio do sistemas, como a criação de gado, e a quantidade de espécies presentes em cada arranjo, não influenciaram nos fatores edáficos avaliados.

PALAVRAS-CHAVE: Agroflorestas, Arranjo, Compactação.

\section{SOIL DENSITY AND MOISTURE IN AGROFORESTRY SYSTEMS}

ABSTRACT: Agroforestry systems (AFSs) are integrated systems consisting of agricultural and forest species, which seek to maintain natural soil conditions. In this way, the work aimed to evaluate and compare two soil physical attributes, density and humidity, in different agroforestry systems. The soil samples were collected using the volumetric ring 
method, considering the depth of $0-5 \mathrm{~cm}$ in the soil for the determination of the density and humidity. The analyzes were carried out in the nursery area of the Federal University of the West of Pará. The experimental design was completely randomized with six treatments and five replicates. The data were submitted to the normality test, based on the Kruskal Wallis non-parametric test for density, due to non-normality of the data, and the Tukey test for the moisture data. The density found in the AFSs studied ranged from 0.7 to $1.2 \mathrm{~g} \cdot \mathrm{cm}^{-3}$, thus being considered as soils under normal conditions, with no compaction levels. Among the systems studied, AFS 4 had a higher density level and lower moisture content and AFS 3 presented better conditions with higher moisture value and lower density. The pre-planting history of the systems, such as cattle breeding, and the number of species present in each arrangement, apparently did not influence the edaphic factors evaluated.

KEYWORDS: Agroforestry, Arrangement, Compression.

\section{DENSIDAD Y HUMEDAD DEL SUELO EN SISTEMAS AGROFORESTALES}

RESUMEN: Los sistemas agroforestales (SAFs) son sistemas integrados constituidos por especies agrícolas y forestales, los cuales buscan mantener las condiciones naturales del suelo. De este modo, el trabajo objetivó evaluar y comparar dos atributos físicos del suelo, densidad y humedad, en diferentes sistemas agroforestales. El estudio fue realizado en el municipio de Monte Alegre - Pará en una colecta puntual en junio de 2017. Las muestras de suelo fueron recolectadas utilizando el método del anillo volumétrico, considerando la profundidad de 0-5 cm en el suelo para la determinación de la densidad y humedad. Los análisis se realizaron en el área de clasificación del vivero de la Universidad Federal del Oeste de Pará. El delineamiento experimental fue completamente casualizado con seis tratamientos y cinco repeticiones. Los datos fueron sometidos a la prueba de normalidad, y con base en él realizó la prueba no paramétrica de Kruskal Wallis para la densidad, debido a la no normalidad de los datos, y la prueba de Tukey para los datos de humedad. La densidad encontrada en los SAFS estudiados varió de 0,7 a 1,2 g. $\mathrm{cm}^{-3}$, siendo así considerados suelos en condiciones normales, no presentando niveles de compactación. Entre los sistemas estudiados, el SAF 4 presentó mayor nivel de densidad y menor contenido de humedad y el SAF 3 presentó mejores condiciones con mayor valor de humedad y menor de densidad. Aparentemente el histórico anterior a la plantación de los sistemas, como la cría de ganado, y la cantidad de especies presentes en cada arreglo, no influenciaron en los factores edáficos evaluados.

PALABRAS CLAVE: Agroforestación, Arreglo, Compactación. 
Os sistemas agroflorestais são considerados uma das formas mais antigas de uso do solo, apesar de receberem maior atenção somente nos últimos anos (RIGHI e BERNARDES, 2015). Nestes sistemas é empregada a combinação intencionada e integrada entre plantas lenhosas perenes e cultivos agrícolas e/ou animais numa mesma unidade de área, de forma simultânea ou rotacional, proporcionando a agregação de valores socioeconômico-culturais e ambientais, uma vez que os mesmos apresentam potencial para constituírem uma modalidade sustentável de uso e manejo dos recursos naturais (SILVA et al., 2012; RIGHI; BERNARDES, 2015).

Os SAFs são considerados um sistema de manejo que tentam manter as condições do solo mais similares das condições naturais, buscando equilíbrio e otimização do tempo e do espaço (AGUIAR, 2008). Sendo uma prática indicada para a recuperação e conservação das áreas do entorno de reservas ambientais, contribuindo para a melhoria das funções fundamentais que mantém a sustentabilidade do agroecossistema (SILVA et al., 2012).

Diante do uso intensivo da terra, a preocupação com a qualidade e manutenção do solo tem aumentado. Para Borges (2016) sua densidade e seu espaço poroso podem ser utilizados como indicadores da qualidade de acordo com o manejo a que o mesmo está sendo submetido. A densidade é um fator importante por indicar o estado de conservação e principalmente por ter influências em outras propriedades edáficas como infiltração e retenção de água, desenvolvimento de raízes, trocas gasosas e suscetibilidade desse solo aos processos erosivos, além de ser utilizada na avaliação da compactação e/ou adensamento dos mesmos (GUARIZ et al., 2009).

Outro fator importante é a umidade, que também é influenciada pela vegetação, que atua em processos importantes no solo e na planta, como: movimento de água, compactação, aeração e desenvolvimento radicular; tornando, de extrema relevância, 
questões referentes à variabilidade espacial e temporal de umidade para diferentes períodos do ano e em diferentes fases de desenvolvimento de determinadas culturas (GUARIZ et al., 2009). Assim o presente trabalho teve como objetivo avaliar e comparar dois atributos físicos do solo, densidade e umidade, em diferentes SAFs no município de Monte Alegre, Pará.

A área de estudo situa-se no município de Monte Alegre, sede municipal localizada nas coordenadas geográficas 2000'30" de latitude S e 540.'13" de longitude W. Gr., pertencente ao Estado do Pará. A média da temperatura para o período estudado (Junho de 2017 ) foi de $27,6^{\circ} \mathrm{C}$ (máximas de $28,8^{\circ} \mathrm{C}$ e mínimas de 22,5 ${ }^{\circ}$ C) com precipitação de $149 \mathrm{~mm}$ de acordo com os dados disponibilizados pelo Instituto Nacional de Meteorologia (INMET, 2017). O tipo de solo da área são constituídos predominantemente pelo Latossolo Bruno Avermelhado (terra roxa estruturada) e Argissolo Vermelho Amarelo (FAPESPA, 2016).
A cobertura vegetal é representada por espécies que acompanham, generamente, as condições do relevo do território, nas áreas de terra firme, onde encontra-se a Mata Equatorial Amazônica, embora com espécies de menor porte e formações secundárias ou capoeiras em diversos estágios. Apresenta clima tipo Am, da classificação de Kôppen, caracterizada por uma estação seca de pouca duração e umidade elevada. As precipitações pluviométricas, com cerca de 1.969 mm, apresentam distribuição irregular durante o ano, a estação mais chuvosa é a que vai de dezembro a junho, sendo março o mês mais chuvoso e a estação menos chuvosa é a que compreende os meses de julho a dezembro. O período de excesso de água no solo vai de fevereiro a julho (FAPESPA, 2016).

O solo avaliado neste trabalho foi coletado em área onde estão estabelecidos diferentes SAFs que tem suas principais características informadas na Tabela 1. 
Tabela 1. Histórico dos sistemas agroflorestais.

\begin{tabular}{|c|c|c|c|}
\hline SAFS & \begin{tabular}{|l|} 
Ano de \\
implantação
\end{tabular} & Espécies no arranjo & Informações gerais \\
\hline $\begin{array}{c}\text { SAF } \\
2\end{array}$ & 2001 & $\begin{array}{l}\text { Pimenta (Piper nigrum), mamão } \\
\text { (Carica papaya), café (Coffea arábica } \\
\text { L.), mogno africano (Khaya } \\
\text { Ivorensis), mogno brasileiro } \\
\text { (Swietenia macrophylla) e teca } \\
\text { (Tectona grandis). }\end{array}$ & $\begin{array}{l}\text { Implantação da Pimenta e do mamão } \\
\text { no ano de } 2008 \text {. } \\
\text { Café ainda está inserido no sistema, } \\
\text { mas está improdutivo. } \\
\text { A limpeza da área é feita de } 3 \text { em } 3 \\
\text { meses através de roçagem } \\
\text { mecanizada }\end{array}$ \\
\hline $\begin{array}{c}\text { SAF } \\
3\end{array}$ & 2004 & $\begin{array}{l}\text { Teca (Tectona grandis), graviola } \\
\text { (Annona muricata), limão (Citrus } \\
\text { limon), laranja (Citrus sinensis L.) e } \\
\text { pocã (Citrus reticulata). }\end{array}$ & $\begin{array}{l}\text { Ocorre doença na graviola (broca no } \\
\text { fruto). } \\
\text { Aplicação de adubação orgânica } \\
\text { (esterco animal) no cultivo de limão. }\end{array}$ \\
\hline $\begin{array}{c}\text { SAF } \\
4\end{array}$ & 2005 & $\begin{array}{l}\text { Urucum (Bixa orellana), teca } \\
\text { (Tectona grandis), mogno africano } \\
\text { (Khaya Ivorensis), pupunha (Bactris } \\
\text { gasipaes) e laranja (Citrus sinensis L.) }\end{array}$ & $\begin{array}{l}\text { Laranja e pupunha ainda estão em } \\
\text { produção, sendo a pupunha } \\
\text { comercializada na região. O mogno } \\
\text { africano produziu sementes depois de } \\
10 \text { anos de plantio. É feito poda e } \\
\text { roçagem na área. } \\
\text { Aplicação de adubação orgânica } \\
\text { (esterco animal) no cultivo da laranja e } \\
\text { urucum. }\end{array}$ \\
\hline $\begin{array}{c}\text { SAF } \\
5\end{array}$ & 2004 & $\begin{array}{l}\text { Mogno africano (Khaya Ivorensis), } \\
\text { mogno brasileiro (Swietenia } \\
\text { macrophylla), teca (Tectona grandis), } \\
\text { manga (Mangifera indica) e } \\
\text { pupunha (Bactris gasipaes). }\end{array}$ & $\begin{array}{l}\text { A manga e a pupunha encontram-se } \\
\text { em produção. } \\
\text { Para aumentar a produção da manga } \\
\text { é feita poda (abertura) no meio da } \\
\text { arvore. }\end{array}$ \\
\hline $\begin{array}{c}\text { SAF } \\
6\end{array}$ & 2007 & $\begin{array}{l}\text { Mogno africano (Khaya Ivorensis), } \\
\text { cumaru (Dipteryx odorata), bacuri } \\
\text { (Platonia insignis) e pupunha (Bactris } \\
\text { gasipaes). }\end{array}$ & $\begin{array}{l}\text { Área com histórico de pecuária e } \\
\text { plantação de pimenta. }\end{array}$ \\
\hline $\begin{array}{c}\text { SAF } \\
7\end{array}$ & 2008 & $\begin{array}{l}\text { Seringa (Hevea brasiliensis), cupuaçu } \\
\text { (Theobroma grandiflorum), mogno } \\
\text { brasileiro (Swietenia macrophylla) e } \\
\text { teca (Tectona grandis). }\end{array}$ & $\begin{array}{l}\text { É realizada a poda nas árvores de } \\
\text { cupuaçu para retirar a vassoura-de- } \\
\text { bruxa. } \\
\text { Até o ano } 2016 \text { era extraído o látex da } \\
\text { seringueira. }\end{array}$ \\
\hline
\end{tabular}

Para se obter as amostras de solo seguiu os procedimentos de campo de acordo com as orientações de Donagema et al. (2011), utilizando o "método do anel volumétrico". A determinação da densidade e umidade do solo foi realizada de acordo com o Manual de Métodos de Análise de Solo da Embrapaı que consiste em pesar a massa de solo 
úmido (Mu) e em seguida secá-lo em estufa a $105^{\circ} \mathrm{C}$ por 24 horas, e após determinar sua massa seca (Ms). Para ambas variáveis avaliadas considerouse a profundidade de $5 \mathrm{~cm}$ no solo. As análises foram realizadas na área de triagem do viveiro da Universidade Federal do Oeste do Pará.

O delineamento experimental foi inteiramente casualizado com seis tratamentos e cinco repetições. Foi realizado o teste de normalidade para os dados, e devido aos dados de densidade não apresentarem normalidade foi realizado o teste não paramétrico de Kruskal Wallis e para a umidade, que apresentaram normalidade, comparou-se as médias pelo teste de Tukey, utilizando o software Assistat ®versão 7.7 (2017).

A densidade encontrada nos SAFs estudados variou de 0,7 a 1,2 g. $\mathrm{cm}^{-3}$, sendo que os SAFs 3 e 4 diferem estatisticamente entre si, porém são iguais estatisticamente aos outros sistemas (Tabela 2). Os solos destes SAFs se encontram em condições normais, não apresentando níveis de compactação, de acordo com Camargo e Alleoni (2006).

Tabela 2. Densidade do solo e umidade gravimétrica na profundidade de $5 \mathrm{~cm}$ em sistemas agroflorestais no município de Monte Alegre, Pará.

\begin{tabular}{c|c|c}
\hline Área & Densidade $\left(\mathrm{g} \cdot \mathrm{cm}^{-3}\right)$ & Umidade (\%) \\
\hline SAF 2 & $1,1 \mathrm{ab}$ & $11,1 \mathrm{ab}$ \\
\hline SAF 3 & $0,7 \mathrm{a}$ & $16,4 \mathrm{a}$ \\
\hline SAF 4 & $1,2 \mathrm{~b}$ & $6,8 \mathrm{~b}$ \\
\hline SAF 5 & $1,1 \mathrm{ab}$ & $8,3 \mathrm{~b}$ \\
\hline SAF 6 & $1,1 \mathrm{ab}$ & $10,8 \mathrm{ab}$ \\
\hline SAF 7 & $1,1 \mathrm{ab}$ & $10,8 \mathrm{ab}$ \\
\hline
\end{tabular}

*Valores seguidos da mesma letra, na coluna, não possuem diferença significativa ao nível de $5 \%$ de probabilidade no teste Kruskal Wallis para densidade e de Tukey na umidade.

O SAF 4 apresentou maior valor de densidade $\left(1,2 \mathrm{~g} \cdot \mathrm{cm}^{-3}\right)$ e menor teor de umidade $(6,8 \%)$, sendo que os solos com maior densidade possuem uma menor capacidade de retenção de água de acordo com Jesus et al. (2015) e o mesmo acontece na situação inversa, como no SAF 3. 
No SAF 6 houve histórico de pecuária na área, porém este fator parece não ter influenciado na densidade e umidade do solo em comparação a maior parte dos outros sistemas (Tabela 2), assemelhando-se com o encontrado por Bortolini et al. (2016), onde o pisoteio dos animais não influenciou na dinâmica da água.

Com relação à umidade do solo, o SAF 3 apresentou o maior índice (16,4\%) e isso pode ser devido a maior quantidade de matéria orgânica, pois nesta área a coloração do solo é mais escura, apesar deste atributo não ter sido avaliado em laboratório para a área em questão. Neste sentido Costa et al. (2016) afirma que a matéria orgânica proporciona melhor retenção de água pelo solo e, consequentemente a um aumento nos teores de água no solo.

Os SAFs 4 e 5 apresentam os valores mais baixos para a umidade e tem em comum, em sua composição, as espécies mogno africano, pupunha e teca. $\bigcirc$ valor da umidade apresentado no SAF 2 não diferiu estatísticamente do SAF 3, apesar de também possuir a espécie teca em sua composição.

O SAF 3 apresentou as melhores condições de densidade e umidade do solo, e atribui-se este fator devido a presença da matéria orgânica, pela sua coloração escura, que promove boas condições físicas ao solo. No SAF 6, onde ocorreu a criação de gado anterior a implantação do SAF, a atividade não gerou aumento na compactação do solo.

A quantidade de espécies que compõem os arranjos nos sistemas agroflorestais avaliados, não influenciou nos valores de densidade e umidade no solo até a profundidade de $5 \mathrm{~cm}$.

Os atributos do solo avaliados neste trabalho, aliados as informações sobre composição dos SAFs, histórico de uso da terra e o período de cultivo, podem ser utilizados para elaboração de estudos a longo prazo que venham a indicar impactos de cada arranjo nas condições edáficas da área.

\section{REFERÊNCIAS}

AGUIAR, M. I. de. Qualidade física do solo em sistemas agroflorestais, Viçosa 2008. 
89 f. Dissertação (Mestrado em Solos e Nutrição de Plantas) - Universidade Federal de Viçosa, Viçosa, 2008.

BORGES, W. L. B. Alterações físicas em Latossolos cultivados com plantas de cobertura em rotação com soja e milho.

Revista Brasileira de Ciências Agrarias, v. 11, p.149 - 155, 2016.

BORTOLINI, D.; ALBUQUERQUE, J. A.; RECH, C.; MAFRA, Á. L.; RIBEIRO FILHO, H. M. N.; PÉRTILE, P. Propriedades físicas do solo em sistema de integração lavoura-pecuária em Cambissolo Húmico.

Revista de Ciências Agroveterinárias, v. 15, p.60 - 67, 2016.

CAMARGO de, O. A.; ALLEONI, L. R. F. Reconhecimento e medida da compactação do solo. 2006. Disponível em:

<http://www.infobibos.com/Artigos/2006

_2/C6/Index.htm> . Acesso em: 11 set. 2017.

COSTA, C. D. de O.; ALVES, M. C.; SOUSA, A. de P. Armazenamento de água em dois solos sob diferentes usos e manejos. Revista de Ciências Ambientais, v. 10, p.55- 65, 2016.

DONAGEMA, G. K.; CAMPOS, D. B. de; CALDERANO, S. B.; TEIXEIRA, W. G.; VIANA, J. M. Manual de métodos de análise de solo. Embrapa Solos Documentos (INFOTECA-E), 2011. Disponível em: <http://www.cnps.embrapa.br/publicacoe s/> Acesso em: 15 nov. 2017.
EMBRAPA. Manual de métodos de análise de solo. Rio de Janeiro: Embrapa Solos, 2011, v. 2, 230 p.

FAPESPA. Estatísticas Municipais Paraenses:Monte Alegre. Belém: Fundação Amazônia de Amparo a Estudos e Pesquisas, 2016, v. 1, 63 p.

GUARIZ, H. R; CAMPANHARO, W. A; PICOLI, M. H. Si CECÍlIO, R. A; HOLLANDA, M. P. Variação da umidade e da densidade do solo sob diferentes coberturas vegetais. Revista Brasileira de Agroecologia, v. 4, p.3293 - 3296, 2009.

Instituto Nacional de Meteorologia (INMET). Disponível em: $<$ http://www.inmet.gov.br/sim/abre_grafi cos.php>. Acesso em: 10 set. 2017.

JESUS, G. L. de; SILVA, I. R.; ALMEIDA, L. J. F.; DOS SANTOS, M. A.; LEITE, F. P.; NEVES, J. C. L. Produtividade do eucalipto, atributos Físicos do solo e Frações da Matéria orgânica influenciadas pela intensidade de tráfego e resíduos de colheita. Revista Brasileira de Ciência do Solo, v. 39, p.1190 - 1203, 2015.

RIGHI, C. A.; BERNARDES, M. S. Sistemas Agroflorestais: definição e perspectivas. Piracicaba: Série Difusão, 2015, v. 1, 79 p.

SILVA, M. S. B de; SILVA, E. M. R. D.; PEREIRA, M. G.; SILVA, C. F. D. Estoque de serapilheira e atividade microbiana em solo sob sistemas agroflorestais. Floresta e ambiente, v. 4, p.431 - 441, 2012. 